\title{
In Situ Electron Holography of Ferroelectric Thin Films
}

Myung-Geun $\operatorname{Han}^{1}$, Matthew S. J. Marshall ${ }^{2}$, Lijun Wu${ }^{1}$, Frederick J. Walker ${ }^{3}$, Charles H. Ahn ${ }^{3}$ and Yimei $\mathrm{Zhu}^{1}$

${ }^{1 .}$ Condensed Matter Physics \& Materials Science, Brookhaven National Laboratory, Upton, NY, USA

2. Department of Applied Physics and Center for Research on Interface Structures and Phenomena, Yale University, New Haven, CT, USA

Ferroelectric thin films have received intense research interests because of their switchable spontaneous polarization that can be utilized in technologically important applications, such as nonvolatile data storage and solar energy harvest [1]. However, size and shape of domains strongly depend on electrical and mechanical boundary conditions and are difficult to predict by general thermodynamic theory [2]. The domain structures and their switching properties can be best understood by directly visualizing domains (walls) during switching and characterizing the electrostatic potentials. We have utilized various electron microcopy techniques combined with in situ electrical biasing to study polarization reversal in epitaxial $\mathrm{Pb}\left(\mathrm{Zr}_{0.2} \mathrm{Ti}_{08}\right) \mathrm{O}_{3}$ (PZT) thin films grown on the (001) Nb-doped $\mathrm{SrTiO}_{3}$ (Nb-STO) substrate [3]. TEM samples in a capacitor form are prepared by FIB lift-out and milling. Electrical contacts have been made using piezo-controlled metallic probes (Nanofactory Instruments, $\mathrm{AB}$ ).

We have directly observed ferroelectric domain switching process under various external biases using dark-field TEM imaging (Fig. 1a-c) by tilting the sample to a two-beam condition in order to maximize the $180^{\circ}$ domain contrast resulted from the violation of Friedel's law [4]. Surprisingly, head-to-head domain walls were found in as-grown samples and the domains near the PZT/Nb-STO interface were not switchable. Off-axis electron holography results showed unidirectional potential barrier and electric fields (Fig. 1d and e) across the PZT/Nb-STO interface. These were confirmed by EBIC showing strong electric fields (Fig. 1k-m) found near the interface under positive external biases applied to the Nb-STO substrate.

The observed unidirectional electric fields can be explained by considering band bending and corresponding charge distributions near the PZT/Nb-STO interface. The presence of polarization charge will influence the zero-bias band bending (Fig. 1f) model. Conceptually, the polarization charge can be treated as an external bias to induce the same amount of charge within the ferroelectric. For the negative polarization charge near the PZT/Nb-STO interface can be considered as a forward biasing the heterojunction, thus reducing the band bending (Fig. 1g). This scenario of flattening band bending by polarization implies that the PZT/Nb-STO interface behaves as a pn junction. When a forward bias is applied, the band bending is further reduced, yielding no significant electric fields that can initiate domain switching. As a result, in the course of domain switching, energetically unfavorable charged domain walls with head-to-head configuration are induced in the PZT thin films. The formation of charged domain walls reported in this study can be an origin for imprint and retention loss in ferroelectric thin films, major obstacles for practical applications.

References:

[1] Setter, N. et al, Journal of Applied Physics, 100 (2006).

[2] Catalan, G. et al, Review of Modern Physics, 84 (2012). 
[3] Han, M.-G. et al, Nature Communications, 5 (2014).

[4] Blank, H. et al, Applied Physics Letters 2, 140 (1963).

[5] The authors acknowledge funding from Division of Materials Science and Engineering, Office of Basic Energy Science, the U.S. Department of Energy, under Contract number DE-AC02-98CH10886 and NSF, MRSEC under DMR11926 (CRISP) and DMR 1309868. Dr. Toshihiro Aoki and Dr. Ray Twesten are acknowledged for their useful discussion and helps on electron microscopy.
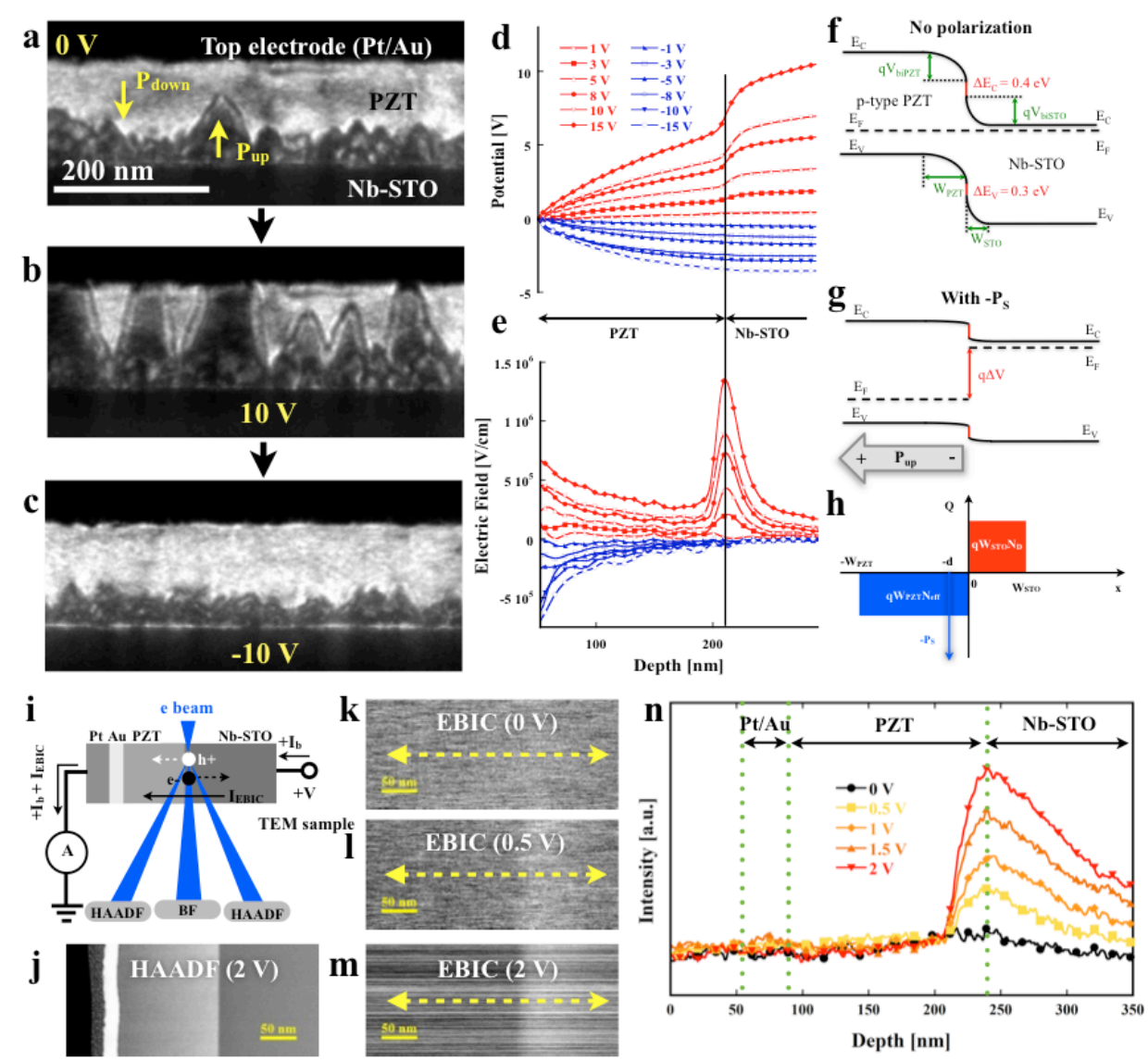

Figure 1. Dark-field TEM images showing ferroelectric domain switching in PZT thin film: as grown (a), after $10 \mathrm{~V}(\mathrm{~b})$, and after $-10 \mathrm{~V}$, respectively. Electrostatic potential line profiles (d) and electric fields along the c axis (e) obtained by electron holography. Schematic band diagrams without (f) and with (g) negative polarization charge $\left(-\mathrm{P}_{\mathrm{s}}\right)$ at the PZT/Nb-STO interface and corresponding charge distributions (h). Schematic (i) of simultaneous acquisition of electron-beam-induced-current (EBIC) images $(k \sim m)$ with HAADF STEM image $(j)$. Line profiles of EBIC $(n)$ showing electric field distributions that are consistent with holography data (e). 